\title{
2, 6-二亚苄基环己酮液晶化合物的合成及性能研究
}

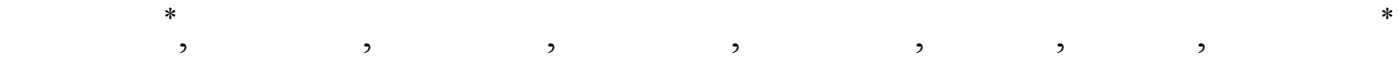 \\ (1) 聊城大学化学化工学院, 聊城 252059 ; \\ (2) 山东大学胶体与界面化学教育部重点实验室, 济南 250100 \\ * 联系人, E-mail: weixilian@126.com, coliw@,sdu.edu.cn \\ 2008-09-24 收稿, 2009-01-01 接受 \\ 国家自然科学基金(批准号: 20673050)和山东省自然科学基金(批准号: 2006B05)资助项目
}

摘要 以苯甲醛、环己酮为原料, 四丁基溴化铵为相转移催化剂, 在氢氧化钠溶液中合成 了具有液晶性质的化合物 2,6-二亚芐基环己酮. 考察了反应时间、温度、碱浓度对产率的 影响. 产品经红外光谱、核磁共振氢谱以及元素分析予以表征, 用差示扫描量热(DSC)、 偏光显微镜和流变仪研究了其液晶行为, 证明为近晶相热致液晶. 对化合物晶体进行了 $\mathrm{X}$ 射线单晶衍射测定, 证实属于单斜晶系, 空间群 $\mathrm{P} 2(1) / \mathrm{c}, a=9.586(1) \AA, b=18.391(2) \AA$, $c=9.433(1) \AA, \alpha=90^{\circ}, \beta=115.816(2)^{\circ}, \gamma=90^{\circ}, D_{\mathrm{c}}=1.217 \mathrm{~g} \cdot \mathrm{cm}^{-3}, V=1496.9(3) \AA^{-3}, Z=4$.

关键词

2, 6-二亚苄基环己酮

液晶化合物

织构

流变性能

$X$ 射线单晶衍射
自从 1888 年发现液晶现象以来 ${ }^{[1,2]}$, 关于液晶合 成和性能测定的研究大量报道. 尤其是热致液晶在 电子显示方面的应用，使设计、合成和表征新型结构 的液晶化合物成为液晶材料研究中的一个活跃领域 ${ }^{[3 \sim 5]}$. $\alpha, \alpha^{\prime}$-二亚芳基环己酮作为医药、农药等精细化学 品的重要中间体，同时也是合成具有潜在生物活性 的嘧啶的前体, 因此该化合物的合成一直备受关注 [6 12]. 近期的研究发现, 该化合物由于本身的分子结 构特点显示出热致液晶的性质, 有关这方面的研究 还未见报道. 因此对该化合物的性能尤其是液晶行 为的研究具有重要意义.

本课题组在前人工作的基础上 ${ }^{[12]}$, 以苯甲醛和 环己酮为原料，合成了液晶化合物双亚苄基环己酮. 用元素分析、红外光谱、核磁共振等对化合物的分子 结构进行了表征; 用差示扫描量热(DSC)和偏光显微 镜等对其液晶性能进行了测定; 用X射线单晶衍射仪 对得到的化合物单晶进行了结构确定; 同时, 对液晶 的流变性能与结构的关系进行了考察.

\section{1 实验}

(i ) 仪器和试剂. 环己酮(AR, 天津市化工试 剂三厂)、苯甲醛( $\mathrm{AR}$, 天津市精细化工研究所), 未进
一步纯化. 氢氧化钠(AR)；95\%乙醇(AR); 四丁基溴 化铵 (AR, 济宁市化工研究所); 二次蒸馏水. Nicolet460FT-IR 型红外光谱仪(美国 Thermo Nicolet 公司); PE-2400 II 元素分析仪(美国 Perkin Elmer 公司); MP-400 型核磁共振仪(美国 Varian 公司); 偏光显微 镜(XP-10, 南京光学仪器厂); 差示扫描量热仪(Pyris Diamond DSC, 美国 Perkin Elmer 公司); 热重分析 仪(WRT-2P, 上海精密科学仪器有限公司); Bruker Smart 21000 CCD-X 射线衍射仪(美国 Bruker 公司); $\mathrm{AR} 2000 \mathrm{ex}$ 型应力控制流变仪(美国 TA 公司).

(ii) 化合物的合成. 取 $5 \mathrm{~mL}$ 苯甲醛、 $0.1 \mathrm{~g}$ 四 丁基溴化铵及氢氧化钠溶液于四口烧瓶中, 恒温, 用 衡压漏斗慢慢滴加环己酮, 搅拌, 反应 $0.5 \sim 2 \mathrm{~h}$, 反应 完毕将所得产物倒入烧杯, 静置 $1 \mathrm{~d}$, 至黄色粒状固 体大量出现. 经减压抽滤, 先后用冰水冷却的 $95 \%$ 乙 醇和蒸馏水淋洗, 再用冷的 $95 \%$ 乙醇洗涤. 干燥 $24 \mathrm{~h}$, 得到亮黄色颗粒状固体.

(iii) 液晶织构的测定. 取少量合成样品于载玻 片上, 盖好盖玻片, 在带热台的偏光显微镜下观察其 加热和冷却过程的液晶织构, 反复进行多次以确定 其织构, 并拍摄纹理照片.

(iv) 特征温度的测定. 用电子天平准确称量 
$0.0050 \mathrm{~g}$ 合成样品于小坩锅内, 测定 DSC 曲线, 以确 定样品的熔点和清亮点温度, 升温速率为 $5^{\circ} \mathrm{C} / \mathrm{min}$, 氮气保护. 同样方法测定样品的热稳定性.

（v）流变性测量. 在应力控制流变仪上进行流 变性能测量. 雉板直径为 $20 \mathrm{~mm}$, 雉角为 $2^{\circ}$, 板间距 $0.05 \mathrm{~mm}$, 测量温度为 $120 \sim 160^{\circ} \mathrm{C}$. 取适当的应力范 围, 在频率为 $1 \mathrm{~Hz}$ 时进行应力扫描. 测定振荡剪切 过程中模量随应力的变化, 确定样品的线性黏弹区. 在确定的线性黏弹区内取应力点, 进行小振幅振荡 实验, 考察样品的模量随频率变化的关系.

\section{2 结果与讨论}

\section{1 化合物的合成}

根据目标化合物的分子结构, 可利用 2 分子苯甲 醛与 1 分子环己酮发生缩合反应制得. 由于环己酮本 身具有 $\alpha$ 氢, 可能发生自身缩合反应, 降低产率. 因 而本文采用的合成方法为: 以四丁基溴化铵为相转 移催化剂, 将环己酮慢慢滴加到苯甲醛与氢氧化钠 水溶液的混合物中. 反应式如下:

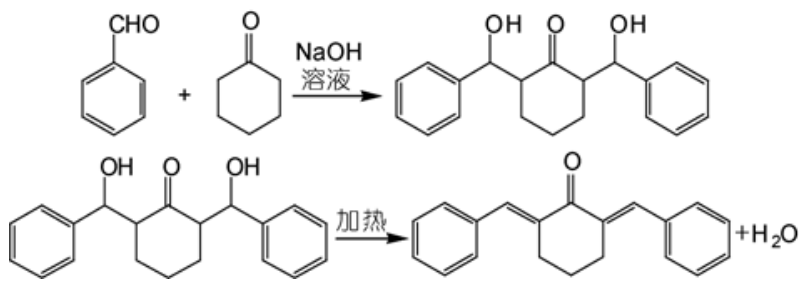

所合成的化合物为亮黄色固体, 不溶于水, 微溶 于乙醇, 易溶于氯仿、乙酸乙酯和丙酮。而环己酮、 苯甲醛与乙醇互溶, 氢氧化钠易溶于水. 因此, 采用 冷的乙醇水溶液洗涤粗产品.

实验表明, 当温度为 $40^{\circ} \mathrm{C}$, 碱浓度为 $15 \%$, 反应 时间为 $30 \mathrm{~min}$ 时, 产率可达 $90 \%$ 以上.

\section{2 化合物结构表征}

液晶化合物 $\left(\mathrm{C}_{20} \mathrm{H}_{18} \mathrm{O}\right)$ 的元素分析结果与理论值吻 合. 测定值 (理论值) 分别为 C $87.50 \%(87.56 \%), \mathrm{H}$ $6.80 \%(6.61 \%)$, O $5.80 \%(5.83 \%)$.

红外光谱数据: 化合物在 $3100 \sim 3010 \mathrm{~cm}^{-1}$ 的吸 收峰为苯环氢与双键氢的特征吸收, $1660 \mathrm{~cm}^{-1}$ 处为 前基和碳碳双键的吸收峰, $1607,1573 \mathrm{~cm}^{-1}$ 及 771 , $695 \mathrm{~cm}^{-1}$ 为苯环的特征吸收.

图 1 为产物的 ${ }^{1} \mathrm{H} \mathrm{NMR}$ 谱图(溶剂为 $\mathrm{CDCl}_{3}, \mathrm{TMS}$ 为内标): $\delta \sim 7.8$ (单峰, $2 \mathrm{H}$ ) 为碳碳双键上的质子峰, $\delta 7.5 \sim 7.2$ (多重峰, $10 \mathrm{H}$ ) 为苯环的质子峰, $\delta \sim 2.9$ (三重

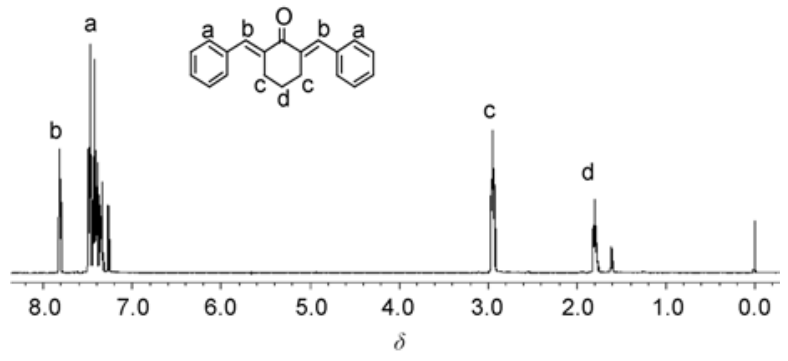

图 1 产物的 ${ }^{1} \mathrm{H}$ NMR 谱图

峰, $4 \mathrm{H}$ )为环己酮 3 位、 5 位上的质子峰, $\delta \sim 1.8$ (五重 峰, $2 \mathrm{H}$ )为环己酮 4 位上的质子峰.

元素分析、红外光谱及核磁共振数据表明, 所合 成的化合物为目标产物.

\section{3 化合物液晶织构确定}

采用Neubert的测量步骤 ${ }^{[13]}$ 来确定液晶的纹理. 在偏光显微镜的正交光系统下观察时, 室温下原始 样品为亮黄色结晶并有晶体的干涉现象, 升温至约 $110^{\circ} \mathrm{C}$ 时开始流动, 呈现出焦雉状纹理织构, 且液晶 颜色由亮黄变为橘黄. 图 2(a)是在约 $125^{\circ} \mathrm{C}$ 时的偏光 显微镜照片. 照片中能看到破碎的焦雉纹理, 参照文 献[5,14 16], 该样品显示为近晶 $\mathrm{C}\left(\mathrm{S}_{\mathrm{C}}\right)$ 相液晶的典型 纹理织构, 这是由于物质中含有手性分子(可由下面 的单晶衍射测定证实). 图 2(b)是温度上升到约 $150^{\circ} \mathrm{C}$ 时的偏光照片, 显示了清晰的焦雉纹理, 这是近晶 $\mathrm{A}$ 相 $\left(\mathrm{S}_{\mathrm{A}}\right.$ 相液晶)的典型织构 $[15]$, 说明样品从熔融后出现 液晶到各向同性的溶液出现, 中间可能经历了从 $\mathrm{S}_{\mathrm{C}}$ 到 $\mathrm{S}_{\mathrm{A}}$ 的转变过程. 从 $210^{\circ} \mathrm{C}$ 开始, 视场中焦锥织构减 少, 到 $260^{\circ} \mathrm{C}$ 以上视野内出现暗区直到分解温度. 有 关样品在加热过程中的相变温度可以由DSC曲线确 定.

另外, 将样品膜在 $210^{\circ} \mathrm{C}$ 以上进行热处理 $5 \mathrm{~min}$, 紧接着淬冷到室温, 最后置于偏光显微镜下进行观 察, 发现样品仍可保持焦雉织构 (见图 2(c)).

\section{4 化合物的 DSC 和热重曲线}

样品的热重分析和差热分析曲线如图 3(a)和(b). 化合物的热稳定性经过 TGA 测试, 其分解温度高于 $300^{\circ} \mathrm{C}$, 因此本文中液晶化合物的偏光织构和 DSC 曲 线图是化合物处于稳定状态下所测得的. 图 3(a)为化 合物的 TGA 曲线.

热致液晶有两个重要参数：熔点 $(m p)$ 和清亮点 $(\mathrm{cp})$, 在熔点与清亮点之间为液晶相区间. 实际应用 中的液晶一般要求液晶材料具备较宽的相变温度范 

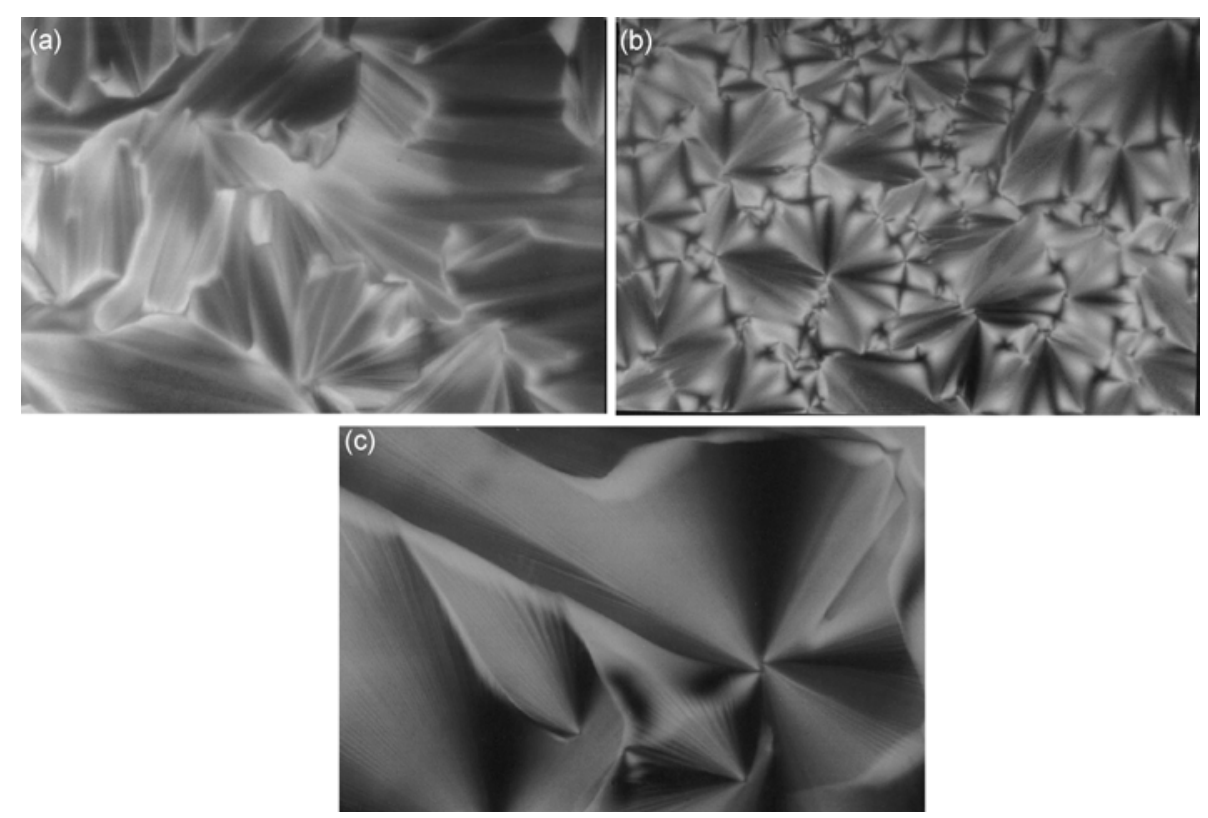

图 2 样品的纹理照片

(a) $\mathrm{S}_{\mathrm{C}}$ 相液晶 $\left(125^{\circ} \mathrm{C}, \times 100\right) ;\left(\right.$ b) $\mathrm{S}_{\mathrm{A}}$ 相液晶 $\left(150^{\circ} \mathrm{C}, \times 100\right)$; (c) 加热到 $210^{\circ} \mathrm{C}$ 以上后淬冷到 $24^{\circ} \mathrm{C}$ 时的照片 $(\times 100)$
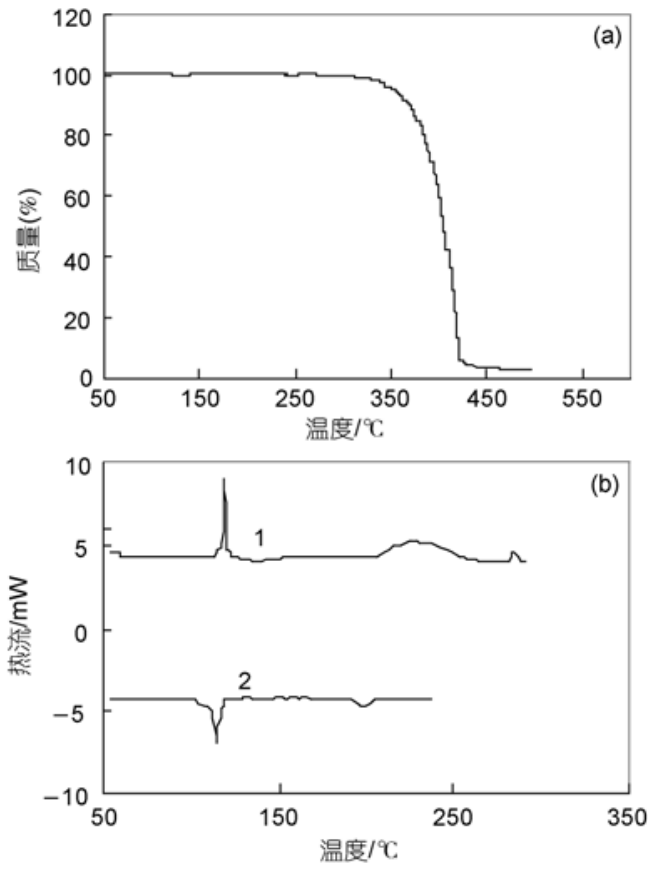

图 3

(a) 液晶化合物的热重分析曲线; (b) 液晶化合物的 DSC 曲线; 1 , 升温曲线; 2 , 降温曲线

围、高物理和化学稳定性、较低的熔点和相变点、低 黏度和较大的各向异性等特点. 由图 3(b)可以看到: 在升温过程中, 曲线分别在 117 和 $210^{\circ} \mathrm{C}$ 处有两个吸 热峰, 第一个吸热峰为样品的熔点峰, 第二个吸热峰
较小为清亮点. 在 $117 \sim 210^{\circ} \mathrm{C}$ 之间为液晶相区间, 在 $210 \sim 255^{\circ} \mathrm{C}$ 之间为液晶转变为各向同性液体的温度范 围, 在此温度区间液晶纹理消失. 另外在DSC曲线中 并未出现由 $\mathrm{S}_{\mathrm{C}}$ 到 $\mathrm{S}_{\mathrm{A}}$ 相明显的相变温度, 只是在液晶 出现以后到 $150^{\circ} \mathrm{C}$ 的范围内看到有很微弱的放热趋势, 这可能是因为从 $\mathrm{S}_{\mathrm{C}}$ 到 $\mathrm{S}_{\mathrm{A}}$ 的相转变能量较小, 一般的 DSC测定很难观察到这个微小的变化 ${ }^{[5,14]}$.

\section{5 化合物晶体结构的测定}

为证实液晶在加热过程中的相变化, 用单晶衍 射仪测定了化合物的单晶结构. 在装有一定量的液 晶化合物的试管中, 加入适量的乙酸乙酯将其溶解. 放置 7 天后即析出亮黄色的块状单晶. 取 $0.50 \mathrm{~mm} \times$ $0.45 \mathrm{~mm} \times 0.43 \mathrm{~mm}$ 大小的晶体置于 CCD X 射线单晶 衍射仪上, 采用石墨单色化的 Mo K $\alpha(\lambda=0.071073 \mathrm{~nm})$ 辐射光源, 在 $2.21^{\circ} \leq \theta \leq 25.01^{\circ}$ 的范围内, 以 $\omega / 2 \theta$ 扫 描方式, 极限指数为 $-10 \leq h \leq 11,-15 \leq k \leq 21,-11 \leq l$ $\leq 10$, 共收集 7392 个衍射点, 其中 2630 个为可观测 点 $[I>2 \sigma(I)]$. 晶体结构由直接法解出, 非氢原子坐标 及各向异性参数用 HELXL-97 程序以最小二乘法对 F2 进行精修. 主要晶体学数据: 单斜晶系, 空间群 $\mathrm{P} 2(1) / \mathrm{c}$, 晶胞参数为 $a=9.586(1) \AA, b=18.391(2) \AA$, $c=9.433(1) \AA, \alpha=90^{\circ}, \beta=115.816(2)^{\circ}, \gamma=90^{\circ}, D_{\mathrm{c}}=$ $1.217 \mathrm{~g} \cdot \mathrm{cm}^{-3}, V=1496.9(3) \mathrm{A}^{-3}, Z=4 . F(000)=584$, $\mu=0.073 \mathrm{~mm}^{-1}$, 最终偏离因子 $R_{1}=0.0383, \mathrm{w} R_{2}=$ 
$0.0838, w=1 /\left[S^{2}\left(F_{\mathrm{o}}{ }^{2}\right)+(0.0328 P)^{2}+0.3516 P\right]$. 其中 $P=$ $\left.F_{\mathrm{o}}{ }^{2}+2 F_{\mathrm{c}}{ }^{2}\right) / 3, S=1.002$, 剩余电子密度的最大正负值 分别为 0.154 和 $-0.112 \mathrm{e} \cdot \AA^{-3}$. 化合物的分子结构、晶 胞中分子的堆积见图 4 和 5 , 部分键长和键角列于表 1. 由化合物晶胞堆积图和表 1 数据, 可知该化合物 分子显示了手性结构, 这与文献[17]相吻合. 由单晶 衍射的测定结果可以很好地解释液晶化合物在加热 过程中的相变. 因为该样品晶体为单斜晶系, 其分子 排列在 $Z$ 轴方向上有一定的夹角, 当样品加热到熔点 后, 样品还基本保持晶体的分子排列, 而 $\mathrm{S}_{\mathrm{C}}$ 相液晶的 结构是分子排列成层, 层内分子长轴相互平行, 其方 向和层面成倾斜排列, 正好符合单斜晶系的分子排 列, 因而在偏光显微镜下显示近晶 $\mathrm{S}_{\mathrm{C}}$ 相的特征纹理. 当温度进一步升高时, 分子运动加剧, 倾斜排列的分 子变得松散, 成为垂直排列, 在偏光下显示了近晶 $\mathrm{S}_{\mathrm{A}}$ 相的焦雉纹理.

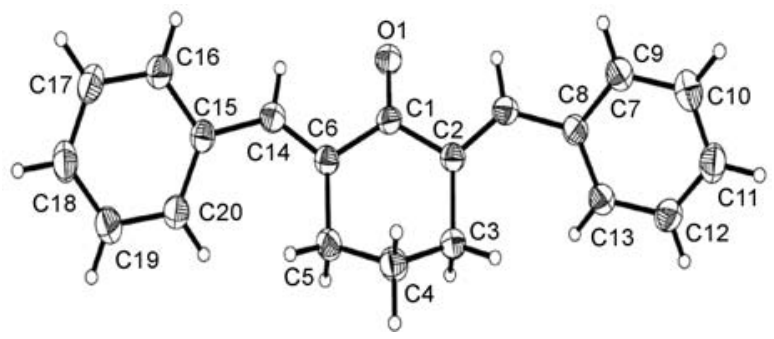

图 4 化合物的分子结构

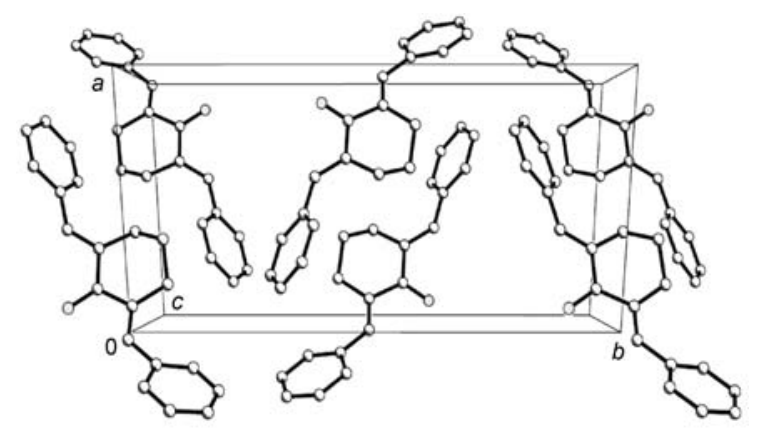

图 5 化合物晶胞堆积图

\section{6 化合物流变性测量}

图 6 为样品在不同温度时的应力扫描图. 应力扫 描中, 复合模量 $\left(\left|G^{*}\right|\right)$ 的平台值可以表示体系中形成 的网络结构的强弱. 可以看出, 样品在 $140^{\circ} \mathrm{C}$ 时结构 强度最大, $125^{\circ} \mathrm{C}$ 的强度最低. 这可能是由于样品刚开 始有固体融化, 还未来得及形成网络结构. 在 130 $140^{\circ} \mathrm{C}$ 之间结构开始发生变化. 也许液晶结构从 $\mathrm{S}_{\mathrm{C}}$ 到 $\mathrm{S}_{\mathrm{A}}$ 的相转变就在此温度间. 即在 $130^{\circ} \mathrm{C}$ 时 $\mathrm{S}_{\mathrm{C}}$ 结构开
表 1 部分键长和键角

\begin{tabular}{|c|c|c|c|}
\hline \multicolumn{2}{|c|}{ 键长/nm } & \multicolumn{2}{|l|}{ 键角 $/\left(^{\circ}\right)$} \\
\hline$C(1)-C(2)$ & $1.495(2)$ & $\mathrm{O}(1)-\mathrm{C}(1)-\mathrm{C}(2)$ & $120.13(15)$ \\
\hline$C(1)-C(6)$ & $1.497(2)$ & $\mathrm{O}(1)-\mathrm{C}(1)-\mathrm{C}(6)$ & $120.66(16)$ \\
\hline$C(2)-C(7)$ & $1.336(2)$ & $C(2)-C(1)-C(6)$ & $119.20(15)$ \\
\hline$C(2)-C(3)$ & $1.500(2)$ & $C(7)-C(2)-C(1)$ & $117.01(15)$ \\
\hline$C(3)-C(4)$ & $1.518(2)$ & $C(7)-C(2)-C(3)$ & $124.94(16)$ \\
\hline$C(4)-C(5)$ & $1.516(3)$ & $C(1)-C(2)-C(3)$ & $117.95(15)$ \\
\hline$C(5)-C(6)$ & $1.506(2)$ & $C(2)-C(3)-C(4)$ & $110.39(14)$ \\
\hline$C(6)-C(14)$ & $1.340(2)$ & $C(5)-C(4)-C(3)$ & $111.58(15)$ \\
\hline$C(7)-C(8)$ & $1.463(2)$ & $C(6)-C(5)-C(4)$ & $112.95(15)$ \\
\hline$C(8)-C(9)$ & $1.388(3)$ & $C(14)-C(6)-C(1)$ & $116.21(15)$ \\
\hline$C(8)-C(13)$ & $1.390(2)$ & $C(14)-C(6)-C(5)$ & $125.07(16)$ \\
\hline $\mathrm{C}(9)-\mathrm{C}(10)$ & $1.375(3)$ & $C(1)-C(6)-C(5)$ & $118.64(15)$ \\
\hline$C(10)-C(11)$ & $1.371(3)$ & $C(2)-C(7)-C(8)$ & $129.70(16)$ \\
\hline$C(11)-C(12)$ & $1.371(3)$ & $C(9)-C(8)-C(13)$ & $117.51(17)$ \\
\hline$C(12)-C(13)$ & $1.377(3)$ & $C(9)-C(8)-C(7)$ & $119.51(16)$ \\
\hline$C(14)-C(15)$ & $1.468(2)$ & $C(13)-C(8)-C(7)$ & $122.93(17)$ \\
\hline$C(15)-C(20)$ & $1.386(2)$ & $\mathrm{C}(10)-\mathrm{C}(9)-\mathrm{C}(8)$ & $121.27(18)$ \\
\hline$C(15)-C(16)$ & $1.394(2)$ & $\mathrm{C}(11)-\mathrm{C}(10)-\mathrm{C}(9)$ & $120.30(2)$ \\
\hline$C(16)-C(17)$ & $1.378(3)$ & $C(10)-C(11)-C(12)$ & $119.30(2)$ \\
\hline$C(17)-C(18)$ & $1.372(3)$ & $\mathrm{C}(11)-\mathrm{C}(12)-\mathrm{C}(13))$ & $120.77(19)$ \\
\hline$C(18)-C(19)$ & $1.376(3)$ & $\mathrm{C}(12)-\mathrm{C}(13)-\mathrm{C}(8)$ & $120.75(19)$ \\
\hline \multirow[t]{9}{*}{$C(19)-C(20)$} & $1.376(2)$ & $C(6)-C(14)-C(15)$ & $131.01(17)$ \\
\hline & & $C(20)-C(15)-C(16)$ & $118.07(16)$ \\
\hline & & $C(20)-C(15)-C(14)$ & $124.84(16)$ \\
\hline & & $C(16)-C(15)-C(14)$ & $117.08(16)$ \\
\hline & & $\mathrm{C}(17)-\mathrm{C}(16)-\mathrm{C}(15)$ & $120.92(18)$ \\
\hline & & $\mathrm{C}(18)-\mathrm{C}(17)-\mathrm{C}(16)$ & $120.09(18)$ \\
\hline & & $\mathrm{C}(17)-\mathrm{C}(18)-\mathrm{C}(19)$ & $119.67(19)$ \\
\hline & & $\mathrm{C}(20)-\mathrm{C}(19)-\mathrm{C}(18)$ & $120.56(19)$ \\
\hline & & $C(19)-C(20)-C(15)$ & $120.60(17)$ \\
\hline
\end{tabular}

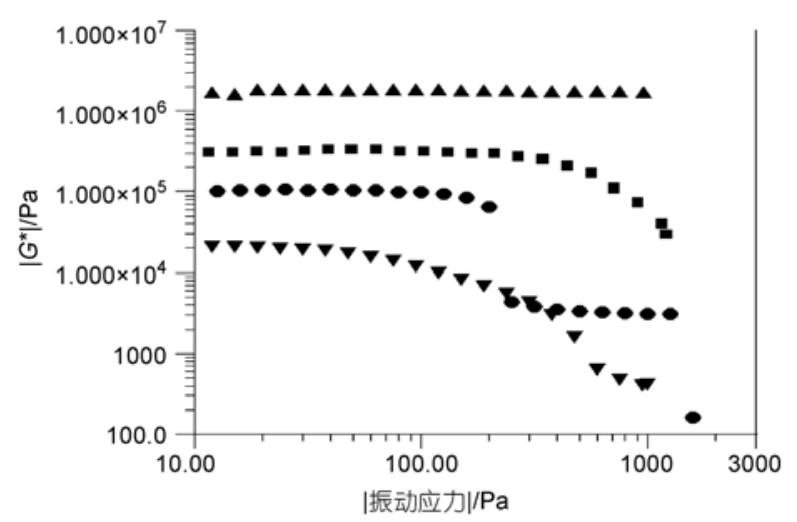

图 6 样品的应力扫描图

$\boldsymbol{\nabla}, 125^{\circ} \mathrm{C} ; \bullet, 130^{\circ} \mathrm{C} ; \boldsymbol{\Delta}, 140^{\circ} \mathrm{C} ; \mathbf{\square}, 160^{\circ} \mathrm{C}$

始发生变化, 到 $140^{\circ} \mathrm{C}$ 已经转变成 $\mathrm{S}_{\mathrm{A}}$ 构型. 因为 $\mathrm{S}_{\mathrm{A}}$ 结 构中分子长轴和层平面垂直, 层厚和分子长度相当 ${ }^{[5]}$, 从理论上分析, 此时的网络强度应该较大, 因此表现 出模量的平台值最大, 之后随着温度的升高逐渐松 
散，模量下降.

进行了切变速率扫描实验, 得到不同温度下样 品复合黏度和剪切速率的关系曲线(图 7). 表明液晶 在实验温度范围内为典型的假塑型流体, 呈现剪切 稀释的性质.

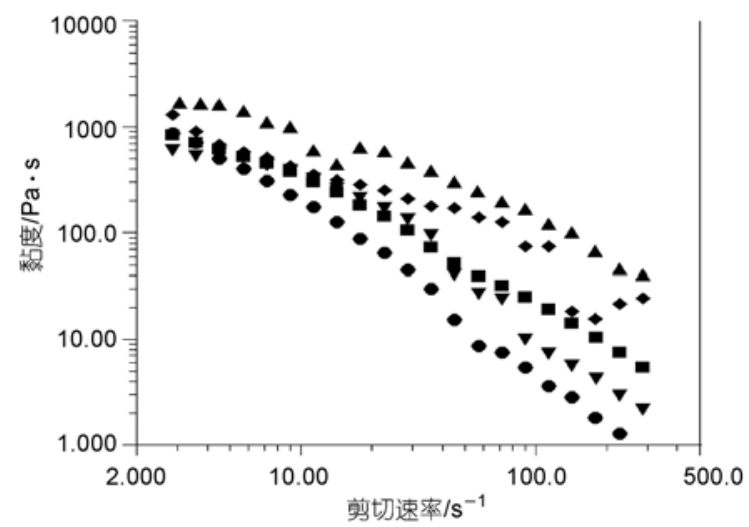

图 7 样品的稳态扫描图

$\boldsymbol{\diamond}, 123^{\circ} \mathrm{C} ; \boldsymbol{\nabla}, 125^{\circ} \mathrm{C} ; \boldsymbol{\bullet}, 130^{\circ} \mathrm{C} ; \boldsymbol{\Delta}, 140^{\circ} \mathrm{C} ; \mathbf{\square}, 160^{\circ} \mathrm{C}$

选择线性黏弹区内的应力值, 进行频率扫描, 得 到不同温度下样品的储能模量 $\left(G^{\prime}\right)$ 、耗能模量 $\left(G^{\prime \prime}\right)$ 随 角频率变化的关系曲线(图 8). 可以看出, 体系各温 度的储能模量基本是一条直线, 不随频率变化, 而耗 能模量规律性较差, 大体上都有一个最小值, 且储能 模量远远高于耗能模量, 两条曲线未出现交叉点. 储 能模量可定性表示体系内形成的网络结构的强弱和 规律性, 可知在此温度范围内, 样品主要表现为弹性, 这也是近晶相液晶的主要特征 ${ }^{[18,19]}$. 从 $120 \sim 130^{\circ} \mathrm{C}$ 三 条直线几乎平行, 说明在这个温度范围内液晶样品 的结构未发生变化, 只是弹性强度随温度的升高而

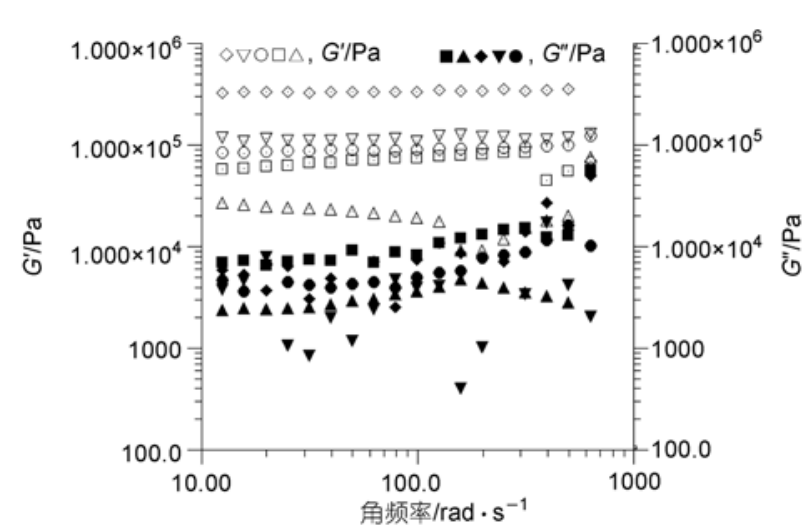

图 8 不同温度下样品的频率扫描图

, $123^{\circ} \mathrm{C} ; \nabla \boldsymbol{\nabla}, 125^{\circ} \mathrm{C} ; \bigcirc \boldsymbol{\bullet}, 130^{\circ} \mathrm{C} ; \triangle \boldsymbol{\Delta}, 140^{\circ} \mathrm{C}$;

, $160^{\circ} \mathrm{C}$

降低. 140 和 $160^{\circ} \mathrm{C}$ 两条直线显示出不同的趋势, 并且 $140^{\circ} \mathrm{C}$ 的弹性模量最低(在图 7 中黏度最大), 也证实 了约在 $130 \sim 140^{\circ} \mathrm{C}$ 之间结构发生了改变.

\section{3 结论}

以环己酮和苯甲醛为原料，合成了双亚苄基环 己酮，用元素分析、红外光谱及核磁共振氢谱等方法 表征了结构. 对化合物进行了性能测试, 发现了该化 合物具有热致液晶特性. 在偏光显微镜下观察, 温度 在 $120^{\circ} \mathrm{C}$ 以上, 液晶织构为近晶 $\mathrm{S}_{\mathrm{C}}$ 相, 到约 $130^{\circ} \mathrm{C}$ 以 上逐渐转化为近晶 $\mathrm{S}_{\mathrm{A}}$ 相. DSC 曲线表明, 样品的熔点 $\left(T_{\mathrm{m}}\right)$ 为 $117^{\circ} \mathrm{C}$, 清亮点 $\left(T_{\mathrm{i}}\right)$ 为 $210^{\circ} \mathrm{C}$, 在 $117 \sim 210^{\circ} \mathrm{C}$ 之 间为液晶相区域. 不同温度下的流变曲线表明, 该样 品在所测定的温度区间, 主要表现为弹性, 频率扫描 证实, 大约在 $130^{\circ} \mathrm{C}$ 以上液晶的微观结构可能由 $\mathrm{S}_{\mathrm{C}}$ 相 转化为 $S_{A}$ 相. $X$ 射线单晶衍射测定证明, 样品属于单 斜晶系，空间群 P2(1)/c.

\section{参考文献}

1 Renitzer F. Beitrage zur kenntiss des cholesterins (Contributions to the understanding of cholesterol). Monatshefte für Chemie(Wien), 1888, (9): 421-441

2 Lahmann O. Uber fliessende krystalle (On flowing crystals). Zeitchrift für Physikalische Chemie, 1889, (4): 462 - 472

3 Sluckin T J, Dunmur D A, Stegemeyer H. Crystals that Flow, Classic Papers from the History of Liquid Crystals. London and New York: Taylor \& Francis, 2004

4 Kong X X, Tang B Z. Synthesis and novel mesomorphic properties of the side-chain liquid crystalline polyacetylenes containing phenyl benzoate mesogens with cyano and methoxy tails. Chem Mater, 1998, 10 (11): 3352-3363[DOI]

5 王良御, 廖松生. 液晶化学. 北京: 科学出版社, 1988. 50-80

6 谷勤翠, 武长城, 陈寿羲, 等. 固化诱导条带织构和焦锥织构装饰技术研究小分子液晶的向错形态. 科学通报, 2002, 47(14): $1071-1073$

7 Hathaway B A. Analdol condensation experiment using number of aldehydes and ketones. J Chem Educ, 1987, 64: 367-368

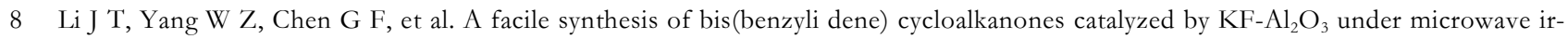


radiation. Synthetic Commun, 2003, 33: 2619-2621 $\underline{\text { [DOI] }}$

Iranpoor N, Kazemi F. RuC ${ }^{13}$ catalyzed aldol condensation of aldehydes and ketones. Tetrahedron, 1998, 54: 9475-9480[DOI]

Nakano T, Migita T. A convenient synthesis of $\alpha, \alpha^{\prime}$-bis(benzylidene) cyclohexanone. Chem Lett, 1993, 12: 2157-2158[DOI]

刘明星, 童庆. $\alpha, \alpha^{\prime}$-二亚苄基环已酮的微波合成. 化学与生物工程, 2005, (6): 21-22

Hathaway B A. An aldol condensation experiment using a number of aldehydes and ketones. J Chem Educ, 1987, 64: 367-368

Neubert M E. Liquid Crystals: Experimental Study of Physical Properties and Phase Transitions. Cambridge: Ambridge University

Press, 2001. 48-49

14 王新久. 液晶光学和液晶显示. 北京: 科学出版社, 2006. 447-465

15 Demus D, Richter L. Textures of Liquid Crystals. New York: Verlag Chemie, 1978

16 魏西莲, 尹宝霖, 李千佐, 等。彩色溶致液晶的研究. 科学通报, 2005, 50: 1454-1458

17 Jia Z C, Jonathan R D. Structures of 2.6-bis(benzylidene)eyelohexanone (III) and 3,5-bis(4-dimethylaminobenzylidene)-1-methyl-4piperidone (IV). Acta Cryst, 1989, C 45: 285-289

\title{
Synthesis of 2,6-bis(benzylidene)cyclohexanone and study of liquid crystal Behavior
}

\author{
WEI XiLian ${ }^{1}$, FU ShiZhou ${ }^{1}$, WANG DaQi $^{1}$, SUN DeZhi $^{1}$, YIN BaoLin $^{1}$, YIN Feng $^{1}$, LIU Jie $^{1}$, \\ \& LI GanZuo ${ }^{2}$ \\ ${ }^{1}$ College of Chemistry and Chemical Engineering, Liaocheng University, Liaocheng 252059, China \\ ${ }^{2}$ Key Laboratory of Colloid and Interface Chemistry, Ministry of Education, Shandong University, Jinan 250100, China
}

The liquid crystalline compound, 2,6-bis(benzylidene)cyclohexanone was synthesized using benzaldehyde and cyclohexanone as raw materials, and tetrabutyl ammonium bromide as phasetransfer catalyst in the solution of sodium hydroxide. Effect of many factors, such as reaction time, reaction temperature and concentration of sodium hydroxide, was studied. The product was confirmed by IR, ${ }^{1} \mathrm{H}$-NMR and element analysis. The liquid crystalline behavior of compound was studied by DSC, optical polarizing micrographs and wideangle X-ray diffraction. The experimental results show that the synthesized compound exhibits typical semectic thermot ropic liquid crystal. Meanwhile, the crystal of compound was determined by X-ray single-crystal diffraction. The crystal of the compound belongs to monoclinic system with space group $\mathrm{P} 2(1) / c, a=9.586(1) \AA, b=18.391(2) \AA, c=$ $9.433(1) \AA, \alpha=90^{\circ}, \beta=115.816(2)^{\circ}, Y=90^{\circ}, D_{c}=1.217 \mathrm{~g} \cdot \mathrm{cm}^{-3}, V=1496.9(3) \AA^{-3}$, and $Z=4$.

2,6-bis(benzylidene)cyclohexanone, liquid crystal intermediate, texture structure, rheological property, X-ray single-crystal diffraction 